\title{
Coleção Entomológica Padre Jesus Santiago Moure: um novo centro de referência para a formação de sistematas de formigas (Hymenoptera: Formicidae) \\ Entomological Collection Padre Jesus Santiago Moure: a new reference center for the formation of ant systematists (Hymenoptera: Formicidae)
}

\author{
Paloma L. Andrade' (1) | Rodrigo M. Feitosa' \\ 'Universidade Federal do Paraná. Curitiba, Paraná, Brasil
}

\begin{abstract}
Resumo: Apresentamos aqui um breve histórico da Coleção Entomológica Padre Jesus Santiago Moure da Universidade Federal do Paraná (DZUP), Curitiba, Brasil, com ênfase na origem e na representatividade de seu recém-expandido acervo mirmecológico. Listamos os principais eventos de expansão, as instalações, os grupos de pesquisa associados e as instituições colaboradoras, essenciais para o estabelecimento do acervo de formigas da DZUP. Finalmente, destacamos o exponencial crescimento deste acervo mirmecológico, que passou de quatro gavetas entomológicas e cerca de 1.000 exemplares depositados, em 2013, para, atualmente, cerca de 360 gavetas e 180.000 exemplares.
\end{abstract}

Palavras-chave: Coleções entomológicas. Curadoria. Mirmecologia.

\begin{abstract}
Here we present a brief historic of the Entomological Collection Padre Jesus Santiago Moure of the Federal University of Paraná (DZUP), Curitiba, Brazil, with emphasis on the origin and coverage of its newly expanded myrmecological collection. We list key events of expansion, facilities descriptions, associated research groups, and collaborating institutions essential for the establishment of the DZUP ant collection. Finally, we highlight the exponential growth of the myrmecological collection, from four entomological drawers and about 1,000 specimens deposited in 2013 to about 360 drawers and 180,000 specimens today.
\end{abstract}

Keywords: Entomological collections. Curation. Myrmecology.

ANDRADE, P. L. \& R. M. FEITOSA, 2020. Coleção Entomológica Padre Jesus Santiago Moure: um novo centro de referência para a formação de sistematas de formigas (Hymenoptera: Formicidae). Boletim do Museu Paraense Emílio Goeldi. Ciências Naturais 15(1): 277-288. DOI: http://doi.org/10.46357/bcnaturais.v15i1.244.

Autora para correspondência: Paloma Leal Andrade. Universidade Federal do Paraná. Programa de Pós-Graduação em Entomologia. Departamento de Zoologia. Centro Politécnico - Jardim das Américas. Caixa postal: 19020. Curitiba, PR, Brasil. CEP 81531-980 (palomaandradebio@gmail.com).

Recebido em 20/12/2019

Aprovado em 03/02/2020

Responsabilidade editorial: Lívia Pires do Prado 


\section{A UNIVERSIDADE FEDERAL DO PARANÁ NO CONTEXTO DE COLEÇÕES BIOLÓGICAS}

As coleções biológicas são essenciais para o desenvolvimento da ciência e constituem-se de organismos, ou de partes desses, que, após o exercício de coleta em trabalhos de campo, são processados e sistematizados para que haja um uso posterior, seja ele voltado para fins científicos, didáticos, particulares ou patrimoniais. Uma coleção biológica representa um banco de dados com o máximo de informações sobre o material que abriga. $\bigcirc$ estudo da biodiversidade está aliado ao desenvolvimento de uma nação, sendo as coleções biológicas um patrimônio de interesse científico, tecnológico e, até mesmo, de segurança nacional. Os dados mantidos nesses conjuntos fornecem informações de distribuição geográfica, biodiversidade, exigências ecológicas, mudanças e influências ambientais e humanas (Camargo et al., 2015 apud Camargo, 2005, 2009).

As coleções biológicas no estado do Paraná são conhecidas internacionalmente devido aos projetos de pesquisa embasados por elas e aos pesquisadores renomados a elas vinculados. $\bigcirc$ mesmo se aplica à principal coleção de insetos do estado, a Coleção Entomológica Padre Jesus Santiago Moure (DZUP).

Jesus Santiago Moure (1912-2010), que dá nome à DZUP, foi um dos mais importantes naturalistas brasileiros, sendo responsável pela fundação desta coleção, abrigada na Universidade Federal do Paraná (UFPR), em Curitiba, Brasil (Figura 1). A UFPR, por sua vez, é a mais antiga universidade federal do país, fundada em 19 de dezembro de 1912. Atualmente, a DZUP é a segunda maior coleção de insetos do Brasil, antecedida apenas pela coleção entomológica do Museu de Zoologia da Universidade São Paulo. Esta segunda posição foi tragicamente herdada do Museu Nacional do Rio de Janeiro, que teve seu acervo entomológico devastado pelo incêndio ocorrido em 2018. A DZUP apresenta, hoje, aproximadamente oito milhões de exemplares de todas as ordens (UFPR, 2019).

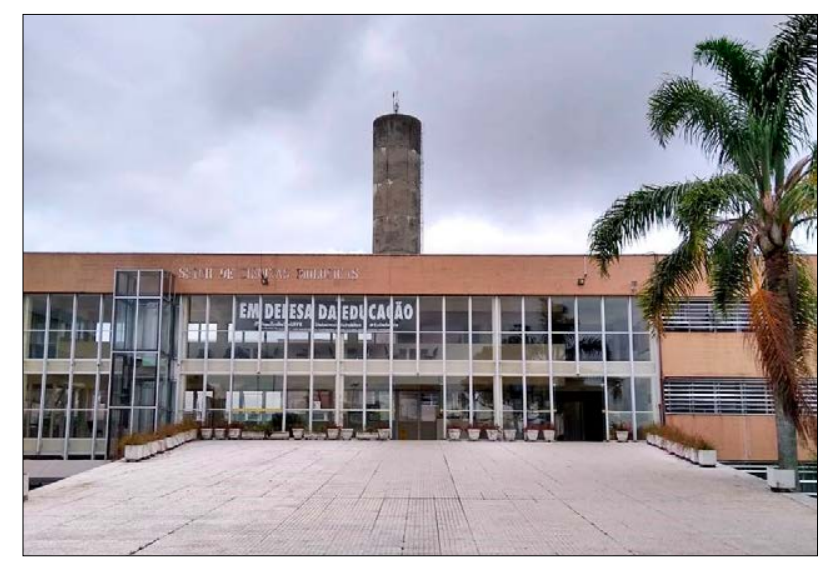

Figura 1. Entrada do setor de Ciências Biológicas da Universidade Federal do Paraná, onde está localizada a Coleção Entomológica Padre Jesus Santiago Moure (DZUP). Foto: Paloma Andrade (2019).

Com uma trajetória intensa e produtiva, Padre Moure, como era conhecido, dedicou boa parte da sua vida ao estudo das abelhas (Hymenoptera: Apidae). Em 1933, o ribeirão-pretano mudou-se para Curitiba, onde começou sua carreira como professor e pesquisador. Pe. Moure foi um dos responsáveis pelo desenvolvimento da Zoologia no Paraná e no Brasil, especialmente após a fundação do Departamento de Zoologia da Universidade Federal do Paraná, o mais antigo do país. Em 1938, foi fundada a Faculdade de Filosofia, Ciências e Letras da UFPR, tendo o Pe. Moure como membro fundador do corpo docente. Apesar de entomólogo, Pe. Moure teve um extenso contato com distintas áreas da Zoologia e foi de grande importância no marco inicial da Biologia Marinha no estado do Paraná em 1951. Isso mostra a sua vocação às Ciências Biológicas e à pesquisa de maneira ampla. Suas colaborações com os professores do Museu Paulista, da Faculdade de Medicina de São Paulo, seus intercâmbios em instituições renomadas e seu cargo como diretor do Museu Paranaense deram a ele todo o conhecimento necessário para tornar-se um impulsionador da pesquisa zoológica no país (Loyola e Silva, 1992).

O Departamento de Zoologia da UFPR é reconhecido pelo nível de excelência em pesquisa científica, com docentes credenciados em um ou mais

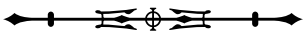


de seus programas de pós-graduação, nominalmente os programas de Entomologia, Zoologia e Ecologia \& Conservação. Considerando-se a produção do corpo docente e a contribuição dos discentes e dos servidores técnico-administrativos, este departamento soma historicamente mais de 2.000 publicações, distribuídas em artigos em periódicos científicos, livros, capítulos de livros, publicações técnicas, relatórios técnico-cientíícos, entre outras, bem como é importante destacar o envolvimento em projetos de extensão (UFPR, 2019).

Esse consolidado grupo apresenta grande repercussão no cenário entomológico internacional nas mais diversas áreas de pesquisa, sendo sua principal linha de atuação Sistemática, Taxonomia e Biogeografia das ordens Diptera, Hymenoptera, Coleoptera, Hemiptera, Lepidoptera, Neuroptera e Odonata. Em adição, projetos em áreas aplicadas com foco em Entomologia Agroflorestal e Médico-Veterinária contribuem para inúmeros estudos em biologia e controle de pragas agrícolas e florestais, ecologia química, genética molecular e manejo integrado de insetos. Já os projetos em Ecologia e Diversidade apresentam ênfase em ecologia evolutiva e etologia das grandes ordens de insetos, além de entomologia forense e diversidade de insetos neotropicais (por exemplo, Rafael et al., 2012; Carvalho \& Almeida, 2011; Moure et al., 2007; Almeida et al., 1998; Baccaro et al., 2015).

Em 2005, o Departamento de Zoologia da UFPR tornou-se a sede do Projeto Taxonline - Rede Paranaense de Coleções Biológicas, sob a coordenação da Dra. Luciane Marinoni, que abrange inúmeras coleções municipais, estaduais, federais e privadas, englobando, além de acervos de todos os grupos zoológicos, diversas coleções microbiológicas e botânicas (Taxonline, 2019). A Coleção Entomológica Padre Jesus Santiago Moure é o ponto central deste projeto, cujo objetivo é a informatização dos dados das coleções para disponibilização online. As informações são obtidas a partir dos rótulos dos exemplares, sendo que esse sistema de armazenamento deverá ser digitalizado continuamente, possibilitando, assim, o rastreamento da origem, da distribuição e da quantidade dos exemplares, além de responder a possíveis outras perguntas envolvendo esses dados. O Projeto Taxonline faz parte, desde 2013, do Sistema de Informações sobre a Biodiversidade Brasileira (SIBBr) e do Global Biodiversity Information Facility (GBIF), os quais são plataformas que disponibilizam dados científicos sobre biodiversidade (Marinoni, L. \& Melo, 2020).

Finalmente, além de seus três programas de pósgraduação de extensa produção e reconhecimento, - Departamento de Zoologia da UFPR é a sede da Sociedade Brasileira de Zoologia e da Sociedade Brasileira de Entomologia e, consequentemente, de suas tradicionais e internacionalmente reconhecidas revistas científicas: a Zoologia - an International Journal for Zoology e a Revista Brasileira de Entomologia - a Journal on Insect Diversity and Evolution.

\section{ORIGEM E EXPANSÃO DO ACERVO DE FORMIGAS DA DZUP}

O acervo de Hymenoptera da Coleção Padre Moure conta com cerca de 600 mil exemplares. Em virtude da especialidade dos renomados melitólogos Padre Moure, Danúncia Urban, Gabriel Melo e, mais recentemente, Rodrigo Gonçalves, membros do Departamento de Zoologia da UFPR, o acervo de Hymenoptera é majoritariamente representado pelas abelhas (Apidae), que somam aproximadamente 340 mil espécimes (SpeciesLink, 2019). Os demais espécimes correspondem principalmente a diferentes grupos de vespas.

No entanto, estas proporções começaram a se alterar a partir de 21 de janeiro de 2013, quando o Dr. Rodrigo Machado Feitosa ingressou como professor do Departamento de Zoologia da UFPR, fundando - Laboratório de Sistemática e Biologia de Formigas da UFPR (LSBF). Sendo a especialidade de Rodrigo a sistemática de Formicidae, ampliou-se rapidamente o estudo de formigas e o enriquecimento do acervo mirmecológico da DZUP. Até então, este acervo contava com algumas amostras históricas e material dos trabalhos

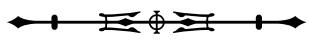


desenvolvidos pelo Laboratório de Dinâmica Evolutiva e Sistemas Complexos, coordenado pelo Dr. Marcio Roberto Pie, membro do Departamento de Zoologia da UFPR e, até então, único pesquisador a conduzir estudos com formigas, na instituição, voltados para ecologia e biologia evolutiva. De fato, a colaboração com o laboratório do Dr. Marcio Pie foi fundamental para o estabelecimento do LSBF, especialmente em projetos envolvendo evolução e biologia molecular.

material histórico referido aqui compreende o acervo coletado e incorporado à coleção antes dos anos 2000. Vários foram os pesquisadores e coletores amadores que depositaram exemplares ou que tiveram suas coleções parcial ou totalmente adquiridas pela DZUP. Nesse sentido, o Coronel Moacyr Alvarenga (1915-2010) é um dos nomes a serem destacados, sendo a sua coleção de insetos representada por aproximadamente 25.000 exemplares na DZUP, principalmente da ordem Coleoptera (Marinoni, R. et al., 1992), embora seja indiscutível sua contribuição para outras ordens. Formigas coletadas por Alvarenga encontram-se bem representadas na DZUP. De fato, o estado do Espírito Santo está representado na DZUP principalmente por espécimes coletados ao final dos anos 1960 por Alvarenga e equipe.

Na década de 1980, teve início o Programa de Desenvolvimento Integrado para o Noroeste do Brasil, financiado pelo Governo Federal, cujo nome abreviado é Polonoroeste. Esse projeto propunha o desenvolvimento econômico em vários setores da agricultura e a construção da BR-364 no Mato Grosso, sendo que o grupo de entomólogos da UFPR ficou responsável pelo levantamento de insetos nas regiões afetadas (Oliveira, R., 1994). Diversas formigas foram coletadas pelo Projeto Polonoroeste e encontram-se hoje depositadas no acervo da DZUP.

No Paraná, as décadas de 1960, 1970 e 1980 foram as mais prolíficas em termos de formigas coletadas no estado e representadas no acervo da DZUP. Em pelo menos dois rótulos da coleção consta o nome do Pe. Moure, sendo um deles referente a uma expedição realizada juntamente com o professor da Olaf Mielke, outro grande nome da entomologia e que ainda está em atividade na Universidade Federal do Paraná. A espécie coletada por Moure e Mielke é uma operária de Dorymyrmex brunneus Forel, 1908, capturada em Foz do Iguaçu, em fevereiro de 1969. O segundo registro em que consta o nome de Pe. Moure refere-se a uma operária de Camponotus rufipes (Fabricius, 1775), coletada no município de Palmas, no Paraná, juntamente com o professor da UFPR e grande especialista em hemípteros Albino Sakakibara, em novembro de 1978. Apesar da existência de diversos eventos avulsos de coleta, a origem de grande parte das formigas no material histórico da coleção para o estado do Paraná é vinculada aos projetos Levantamento da Fauna Entomológica no Estado do Paraná (PROFAUPAR) e Levantamento da Fauna Entomológica do Parque Estadual de Vila Velha, em Ponta Grossa (PROVIVE).

O PROFAUPAR foi realizado durante o período de agosto de 1986 a julho de 1988 em oito localidades do estado do Paraná, com o objetivo de inventariar a entomofauna do estado de forma padronizada com o uso de armadilhas de interceptação de voo do tipo Malaise (Marinoni, R. \& Dutra, 1991). O PROVIVE foi uma iniciativa proposta em 1999 pelos professores Renato C. Marinoni, Luciane Marinoni e Cibele S. Ribeiro-Costa, a qual perdurou até o ano de 2002. As coletas desse projeto foram realizadas ao longo de três anos em áreas do parque, caracterizadas por capões de araucárias e áreas alteradas pela antropização. No caso do PROVIVE, além das armadilhas do tipo Malaise, foram empregadas armadilhas de solo do tipo pitfall.

Embora extremamente importantes para a ampliação do conhecimento entomológico no estado do Paraná, os projetos ora mencionados não tinham como objetivo primário a captura de insetos representantes da fauna edáfica, de modo que formigas não estavam representadas em grande parte das amostras. Uma exceção pode ser feita com relação às formas sexuadas (rainhas aladas e machos), que foram amostradas com maior abundância 
por estes projetos, embora nunca tivessem sido processadas e incluídas no acervo principal até agora. De fato, até o ano de 2013, o acervo de formigas da DZUP contava com apenas quatro gavetas entomológicas completas e aproximadamente 1.000 espécimes oriundos majoritariamente da coleção histórica.

Com o estabelecimento do LSBF, em 2013, as primeiras expedições de coleta e os projetos tinham como objetivo ampliar o conhecimento sobre a mirmecofauna paranaense, um estado surpreendentemente subamostrado para formigas, especialmente em comparação a seus estados vizinhos, São Paulo e Santa Catarina (Kempf, 1972; Ulysséa et al., 2011). Entre março de 2014 e janeiro de 2016, as expedições de coleta do LSBF foram exclusivamente realizadas no estado do Paraná, em diferentes áreas de Mata Atlântica (incluindo campos naturais) e fragmentos de Cerrado. As primeiras coletas foram realizadas na Reserva Natural Rio Cachoeira, hoje Reserva Natural Guaricica, no município de Antonina, na encosta da serra do Mar paranaense (2518' 53,24" S, 48 41' 46,22" O) e no Parque Estadual do Pico do Marumbi, em Piraquara

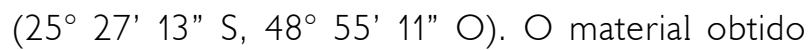
alimentou o primeiro projeto de mestrado desenvolvido no laboratório pela aluna Juliana Maria Calixto, defendido em 2016 (Calixto, 2016).

As expedições seguintes englobaram um projeto maior, aprovado em 2014, intitulado "Formigas e a conservação dos Campos Gerais paranaenses: uma abordagem ecológica e taxonômica", financiado pelo Conselho Nacional de Desenvolvimento Científico e Tecnológico (CNPq), no âmbito do edital Universal. Associado a este estudo também estava o projeto Rede de Pesquisa Biota do Cerrado - Isoptera e Hymenoptera, coordenado pelo Dr. Heraldo Vasconcelos, do Laboratório de Ecologia de Insetos Sociais da Universidade Federal de Uberlândia. As coletas para estes projetos foram realizadas no Parque Estadual de Vila Velha, município de Ponta Grossa (2514' 46" S, $\left.50^{\circ} 01^{\prime} 13^{\prime \prime} \mathrm{O}\right)$, no Parque Estadual do Cerrado, em Jaguariaíva (24 10’ 00" S, 49 39' 56" W), e no Parque Estadual do Guartelá, em Tibagi (24 34' 06" S, $50^{\circ} 16^{\prime} 02$ " O). Essas áreas localizam-se em uma região de fisionomia singular entre os campos naturais brasileiros, denominada de Campos Gerais paranaenses, que se distribuem por $11.761,41$ km² e abrangem 22 municípios paranaenses. Por sua baixa aptidão agrícola, esses campos ainda possuem uma vegetação reliquiar remanescente de épocas mais secas, do período Quaternário. Segundo Melo et al. (2007), os Campos Gerais foram definidos como uma região fitogeográfica, ou seja, caracterizada pela vegetação natural que compreende campos limpos e campos de cerrados naturais, localizados na borda do Segundo Planalto paranaense.

Os dados obtidos nessas expedições embasaram as primeiras pesquisas de iniciação científica do laboratório, conduzidas pelos alunos Weslly Franco e Aline Machado de Oliveira, entre 2014 e 2015 (Franco, 2018; Oliveira, A., 2015). Demais expedições de coleta no estado foram realizadas no Refúgio de Vida Silvestre dos Campos de Palmas, município de Palmas (2631' 40" S, 5136’ 17" O), e no Parque Estadual das Lauráceas, em Adrianópolis e

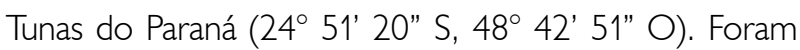
também registradas coletas feitas para fins didáticos (cursos de campo e disciplinas) em áreas dos municípios de Curitiba, Morretes e São José dos Pinhais.

Expedições adicionais foram conduzidas na Reserva Natural Guaricica, no âmbito do Programa de Pesquisas em Biodiversidade (PPBio) Mata Atlântica, coordenado pela Dra. Márcia Marques, do Departamento de Botânica da UFPR, e que passou a integrar o Programa de Pesquisas Ecológicas de Longa Duração (PELD) Lagamar, em uma iniciativa que visa à obtenção de importantes informações para a conservação da biodiversidade e o uso sustentável dos recursos naturais dos ecossistemas brasileiros. Criado em 1996, o PELD tem como foco o estabelecimento de sítios de pesquisa permanentes nos mais diversos biomas e ecossistemas brasileiros integrados em uma rede de colaboração que abrange as mais diversas áreas do conhecimento. As iniciativas do PELD para a gestão 
dos recursos ecossistêmicos trazem retorno através de indicadores de produção em subprojetos, parcerias, recursos humanos, artigos científicos, livros, dissertações, teses, monografias, patentes e programas de pósgraduação (CNPq, 2019).

As coletas do PELD são padronizadas de acordo com a metodologia aplicada para cada grupo. No caso das formigas, o protocolo aplicado foi denominado AntPELD, proposto pelos pesquisadores Rodrigo Feitosa e Márcio Pie, da UFPR, após consulta a colegas ecólogos atuando em diferentes instituições. O protocolo consiste basicamente na instalação de armadilhas de queda do tipo pitfall em três transectos de 500 metros, afastados $1 \mathrm{~km}$ uns dos outros, cada um contendo 20 pitfalls, separados por 25 metros. Este protocolo (AntPELD) foi amplamente divulgado e, atualmente, uma rede de pesquisadores em todos os biomas do Brasil tem realizado coletas no âmbito deste projeto, enviando as formigas capturadas ao acervo da DZUP. A proposta é a realização de diferentes trabalhos sobre a estrutura ecológica e filogenética de comunidades de formigas em variados biomas do Brasil e o estabelecimento de um grande banco de tecidos para estudos envolvendo biologia molecular, sediado na DZUP. Até a presente data, 18 localidades de todos os biomas brasileiros já foram amostradas.

Outra fonte constante de amostras ao acervo de formigas da DZUP é o Curso Formigas do Brasil (CFB), idealizado em consequência da carência de um curso de atualização imersivo em Mirmecologia no país. Coordenado pelos pesquisadores Dr. Fernando Schmidt (Universidade Federal do Acre), Dra. Carla Ribas (Universidade Federal de Lavras) e Dr. Rodrigo Feitosa (UFPR), o curso tem como objetivo proporcionar experiência teórico-prática em distintos aspectos da Mirmecologia a estudantes e profissionais com interesse em formigas. Nesse sentido, - CFB inclui uma etapa intensiva de coleta, com diferentes técnicas de amostragem, e que geram material para fins didáticos e científicos. As edições-piloto foram realizadas em 2012 e 2013, na Universidade Federal de Lavras e na
Universidade Federal de Belo Horizonte, respectivamente. Desde então, o curso passou a ser bianual. Cada edição é realizada em um bioma brasileiro, já tendo passado pelo Cerrado, em 2014 (Uberlândia, Minas Gerais), pela Amazônia, em 2016 (Rio Branco, Acre), e pela Mata Atlântica, em 2018 (Santa Teresa, Espírito Santo). Como dito, o processamento e a incorporação tanto do material do AntPELD como o do CFB são realizados integralmente na DZUP, o que expandiu significativamente o acervo de formigas da coleção.

Em 2014, o LSBF teve seu primeiro projeto temático de grande porte aprovado em âmbito internacional, intitulado Biodiversity Conservation and Scientific Capacity Development in the Brazilian Amazon using Ants as Bioindicators and Ecosystem Health Indicators, com valor global de aproximadamente $R \$ 500.000,00$. Neste projeto, foram conduzidas expedições em regiões de difícil acesso, nunca ou raramente amostradas para formigas no país. Em 2016, foram realizadas coletas no Parque Nacional da Serra das Confusões (Piauí) (9 13' 18” S, $43^{\circ} 29^{\prime} 33^{\prime \prime}$ O), no Parque Nacional da Serra da Capivara (Piauí) (8० 46' 26" S, $42^{\circ} 50^{\prime} 22^{\prime \prime}$ ), na Floresta Nacional Saracá-Taquera (Pará) ( $1^{\circ} 38^{\prime} 31^{\prime \prime}$ S, $56^{\circ} 37^{\prime} 54^{\prime \prime}$ O) e no Parque Nacional da

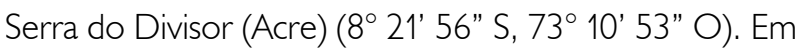
2017, conduzimos expedições ao Parque Nacional das Montanhas do Tumucumaque (Amapá) (200'34” N, $53^{\circ} 32^{\prime} 41^{\prime \prime}$ ) e e ao Parque Nacional do Viruá (Roraima) ( $\left.1^{\circ} 19^{\prime} 50^{\prime \prime} \mathrm{N}, 61^{\circ} 09^{\prime} 05^{\prime \prime} \mathrm{O}\right)$. Finalmente, em 2018 coletamos na Reserva Particular do Patrimônio Natural Serra Bonita (Bahia) (1524' 00" S, $39^{\circ} 34^{\prime}$ 17" O).

Um evento de importância maior na consolidação e na expansão do grupo de pesquisas em Mirmecologia da UFPR e, consequentemente, do acervo de formigas da DZUP foi a contratação do Dr. John E. Lattke pelo Departamento de Zoologia da referida universidade em 2014. John é um dos maiores nomes da sistemática de formigas do mundo, com uma prolífica trajetória científica e passagens profissionais frutíferas pelos Estados Unidos (Davis), Venezuela (Maracay) e Equador (Loja). Ele ainda 
incorporou à DZUP um vasto e precioso lote de formigas em via líquida, coletadas em dezenas de países (em especial, a Venezuela) de todas as regiões biogeográficas do mundo. Trata-se de uma réplica da coleção de formigas do Museo del Instituto de Zoología Agrícola de la Universidad Central de Venezuela, Maracay, Venezuela, da qual John foi curador no período de 1993 a 2014.

Também em 2014, o LSBF recebeu sua primeira grande remessa de material do exterior. A doação foi realizada pelo grupo de pesquisa francês Société Entomologique Antilles-Guyane (SEAG), em atuação na Guiana Francesa. Diversos lotes contendo milhares de formigas coletadas com armadilhas de interceptação de voo de baixa altitude foram enviados à DZUP para depósito. As coletas foram realizadas nas comunas de Saül e Roura, em 2011. Este material, contendo 112 espécies e 22 novos registros para a Guiana Francesa, foi a base para a elaboração da primeira lista de espécies de formigas para a Guiana Francesa (Franco et al., 2019).

Uma vez tendo seu grupo de mirmecólogos estabelecido e três laboratórios em atividade, a DZUP tornou-se muito rapidamente um grande centro de pesquisa em Mirmecologia e uma instituição de referência para o depósito de material-testemunho de pesquisas realizadas com formigas no Brasil e no exterior. São inúmeras as fontes de envio de material à DZUP, incluindo o depósito de vouchers por parte de grupos de pesquisa em ecologia, evolução e comportamento de formigas em mais de 35 instituições no país e no exterior (Feitosa Lab, 2018). Em adição, a DZUP é depositária de material de cursos de campo internacionais, como o Ant Course e o Curso de Hormigas Neotropicales, realizados periodicamente em distintas regiões do mundo, além de projetos de consultoria e de doações de coleções particulares.

O acervo de formigas da Coleção Entomológica Padre Jesus Santiago Moure também se beneficia de diversas colaborações com o Laboratório de Sistemática, Evolução e Biologia de Hymenoptera do Museu de Zoologia da Universidade de São Paulo (MZSP), que tem como curador o Prof. Dr. Carlos Roberto Ferreira Brandão. A coleção de formigas do MZSP é a mais representativa da região Neotropical em número de espécimes-tipo e abrangência geográfica, sendo que a sua história se mescla com a própria história da Mirmecologia neotropical. Os primeiros registros de depósito de material no acervo do MZSP datam do final do século XIX, e o acervo ainda abriga as coleções de grandes nomes históricos da taxonomia de formigas, como os freis franciscanos Tomás Borgmeier (1892-1975) e Walter Kempf (1920-1976) (Klingenberg \& Brandão, 2005).

Além da estreita e frutífera relação com o MZSP, desde o início, o acervo de formigas da DZUP conta com a colaboração do Laboratório de Mirmecologia da Comissão Executiva do Plano da Lavoura Cacaueira (CEPLAC), em Ilhéus, na Bahia, sob a curadoria do Dr. Jacques Delabie. Como suporte para o estabelecimento e a expansão da coleção de formigas da DZUP, entre os anos de 2013 e 2018, o Dr. Jacques Delabie doou ao acervo da DZUP cerca de 2.320 exemplares pertencentes a 906 espécies de formigas de todas as regiões do mundo. Esta doação representa um divisor de águas na história do acervo mirmecológico da DZUP, uma vez que proporcionou independência aos estudos de taxonomia de formigas desenvolvidos na instituição. Além dessa valiosa doação, há uma parceria constante com a CEPLAC por meio de inúmeras visitas para exame de material, colaborações em trabalhos, cursos e afins.

Em 2018, em contato com o Laboratório de Sistemática e Biologia de Formigas da UFPR, o Dr. Jorge L. M. Diniz, da Universidade Federal de Goiás, campus Jataí - um dos maiores nomes da taxonomia de formigas neotropicais e curador de um acervo de formigas de riqueza imensurável -, anunciou sua aposentadoria e, com ela, a proposta de doar integralmente sua coleção à DZUP. Concretizados os trâmites burocráticos, a doação envolveu cerca de 165 gavetas entomológicas e 300 lotes líquidos contendo cerca de 50 mil espécimes das mais diversas regiões biogeográficas do globo, incluindo espécimes-tipo. 
Além de apresentar-se como uma volumosa coleção, o valor científico desta doação está centrado no fato de que este acervo inclui réplicas das coleções particulares do Frei Walter Kempf e do Frei Tomás Borgmeier, representantes máximos da taxonomia de formigas no Brasil e dois dos maiores nomes da Mirmecologia mundial, cujas coleções originais se encontram no Museu de Zoologia da Universidade de São Paulo, como já dito. Ademais, esta doação também envolveu o acervo bibliográfico do laboratório do Dr. Diniz, que conta com publicações históricas, incluindo volumes originais da revista Studia Entomologica, além de separatas, cadernos de campo e catálogos oriundos das bibliotecas de Kempf, Borgmeier e Diniz. Ao todo, o material doado pela Universidade Federal de Goiás representou um incremento de mais de um terço do volume total de espécimes na coleção de formigas da DZUP à época.

Em 2017, um projeto de iniciação científica conduzido pela então graduanda Paloma Andrade (Andrade, 2017) teve como propósito descrever a origem e a representatividade do acervo de formigas da Coleção Entomológica Padre Jesus Santiago Moure, com base nos dados obtidos diretamente das etiquetas dos espécimes e dos outros projetos que geraram o material até o início do ano em questão. Por uma limitação de tempo, foram levantados dados de todas as subfamílias de formigas de ocorrência neotropical, exceto de Agroecomyrmecinae, Martialinae e Myrmicinae. Este estudo foi o primeiro passo para a realização de um grande projeto de informatização do acervo de formigas da DZUP.

Em relação à cobertura geográfica da coleção, o Brasil é, sem dúvidas, o país mais bem representado, tanto em número de espécies quanto em indivíduos coletados, sendo que a DZUP abriga espécimes de todas as regiões geopolíticas e de todos os estados do país. Além do Brasil, a coleção abriga espécimes de mais outros 25 países.

Embora em uma proporção menor do que os estados do Sul e do Sudeste, as regiões Norte e Nordeste são historicamente alvo de expedições científicas. As datas de coleta para o material proveniente da região Nordeste na DZUP variam desde os anos 1990 até 2014. No Norte, Pará e Rondônia apresentam destaque, devido à grande representação no acervo da DZUP de formigas provenientes destes estados, em consequência de grandes expedições realizadas. As formigas da região Norte depositadas nesta coleção estão representadas por espécimes coletados principalmente a partir dos anos 1990, com destaque para amostras que a equipe da pesquisadora Ana Yoshi Harada obteve, a qual conduziu expedições durante vários anos na região. Já os demais estados, incluindo Acre, Amapá e Tocantins, estão representados por formigas coletadas em sua grande maioria entre os anos de 1961 e 1963 por Moacyr Alvarenga e sua equipe. As formigas do estado do Amazonas foram majoritariamente coletadas nos anos 1990 por vários pesquisadores.

Na região Centro-Oeste do Brasil, os estados de Mato Grosso e Mato Grosso do Sul possuem o maior número de espécimes representados na coleção. As localidades de coletas são as mais distintas, incluindo fazendas, reservas e outras áreas de conservação. As expedições também variam muito em relação às equipes e às datas, sendo que os registros de coleta para ambos os estados ocorreram desde o início dos anos 1960 e seguem até os dias atuais. Devido ao projeto Polonoroeste, o estado de Mato Grosso apresenta grande representatividade no acervo. A região Centro-Oeste também se destaca pela Coleção Diniz, que inclui uma ampla série de espécimes coletados em diversas regiões do estado de Goiás.

Para a região Sudeste do Brasil, o estado de Minas Gerais é o que apresenta o maior número de espécimes depositados na DZUP. Isso se deve principalmente ao material-testemunho gerado pelo Curso Formigas do Brasil. As formigas de Minas Gerais presentes na coleção são em grande parte provenientes das edições iniciais do curso, realizadas em diferentes regiões do estado nos anos de 2012 a 2014. Soma-se a este material uma grande quantidade de vouchers depositados na coleção por diversos grupos de pesquisa em ecologia de formigas 
sediados em diferentes instituições e regiões do estado de Minas Gerais, com o maior número de laboratórios desta especialidade no Brasil.

Em relação aos demais estados do Sudeste, São Paulo também é diverso em relação aos coletores e às datas de coleta. Rio de Janeiro está representado por espécies depositadas na DZUP desde o final dos anos 1950. Contudo, a maior parte do material foi coletada no ano de 2015, no projeto conduzido por Chaim Lasmar (Lasmar et al., 2020), então doutorando da Universidade Federal de Lavras, que investigou o efeito da altitude e a formação vegetal sobre a comunidade de formigas na região do Parque Nacional do Itatiaia e depositou os vouchers de centenas de amostras na DZUP. Quanto ao Espírito Santo, grande parte das espécies de formigas depositadas na coleção é proveniente da década de 1960, coletada por Moacyr Alvarenga e equipe, como já mencionado. Contudo, um amplo volume de amostras provenientes da edição de 2018 do Curso Formigas do Brasil, realizado no município serrano de Santa Teresa, foi recentemente incorporado à coleção.

Para o Sul do Brasil, o Paraná, como esperado, conta com o maior número de espécimes depositado na DZUP até o momento. A coleta mais antiga para este estado data de 1966, sem nome de autor ou equipe de coleta. Os exemplares históricos deste estado têm como coletores alguns dos principais nomes da Entomologia brasileira, como Pe. Moure, Olaf Mielke, Albino Sakakibara e Gabriel Melo. O Rio Grande do Sul também está bem representado no acervo, mas com número de espécimes menor em comparação aos estados ao norte dessa região. Santa Catarina está representada por registros de coleta datados desde o final dos anos 1970, mas com numerosas amostras recentes coletadas por Eduardo Cereto (em 2008), Mônica Antunes Ulysséa (em 2009) e Fabiano F. Albertoni (em 2009), então graduandos e pós-graduandos da Universidade Federal de Santa Catarina. Mais recentemente, um imenso lote de material proveniente de Santa Catarina foi depositado na DZUP como parte dos projetos de mestrado e doutorado da pós-graduanda Mila Martins, orientada pelo Dr. Rodrigo Feitosa, e que investiga a fauna subterrânea de formigas em áreas com distintos usos de solo em Santa Catarina e no Paraná (Martins et al., 2020).

Em razão de a coleção DZUP estar situada na América do Sul, este é o continente com maior destaque de material no acervo, incluindo espécimes de praticamente todos os países sul-americanos. Em seguida, em termos de representatividade na coleção, está a Oceania, representada principalmente pela Austrália, por conta de doações feitas pelos pesquisadores Alan Andersen (Charles Darwin University) e Júlio Chaul (Universidade Federal de Viçosa).

\section{ESTADO ATUAL E PERSPECTIVAS PARA O ACERVO DE FORMIGAS DA DZUP}

Até o ano de 2019, o acervo de formigas da Coleção Padre Jesus Santiago Moure contava com 15 subfamílias e, aproximadamente, 170 gêneros de formigas, um número significativo, considerando-se que, no mundo, há hoje 17 subfamílias e 334 gêneros válidos desses insetos (Bolton, 2019). Isto é, o acervo da coleção já abriga representantes de quase todas as subfamílias de formigas conhecidas e metade dos gêneros válidos existentes no mundo, além de parátipos e holótipos de cerca de 60 espécies em mais de dez gêneros.

Além dos mirmecólogos instalados na DZUP, o LSBF recebe constantemente inúmeros visitantes especialistas na taxonomia de diversos grupos de formigas, possibilitando a identificação e a organização dos espécimes do acervo, na maioria das vezes, até o nível específico, o que é de extrema importância para uma coleção de referência. Além disso, o LSBF recebe inúmeros estudantes e pesquisadores do Brasil e do exterior para confirmação e identificação de material de seus projetos. O laboratório é também procurado frequentemente para a realização de estágios para iniciantes em Mirmecologia, especialmente visando à obtenção de prática no processamento de 
amostras e de bases para autonomia em identificação. Esse contato amplia as colaborações e, consequentemente, a representatividade do acervo da DZUP, visto que vouchers de inúmeros estudos são depositados durante essas visitas.

Após sete anos da criação do LSBF, e em decorrência do grande esforço empreendido pelos grupos de pesquisa em Mirmecologia da UFPR, o acervo de formigas da DZUP passou das iniciais quatro gavetas e aproximadamente 1.000 formigas para cerca de 360 gavetas entomológicas e mais de 180.000 exemplares (Figura 2). Sendo a sistemática a principal linha de pesquisa nos laboratórios do Dr. Rodrigo Feitosa e do Dr. John Lattke, diversos estudos envolvendo revisões taxonômicas globais e regionais estão em andamento atualmente, conduzidos por um dos maiores grupos de taxonomistas em formação no mundo hoje, até onde se sabe. Uma vantagem nítida deste cenário consiste no fato de que esses taxonomistas são as autoridades na classificação dos grupos com os quais trabalham, o que se reflete em uma organização extremamente atualizada e precisa do acervo da coleção.

Os trabalhos de revisão taxonômica em andamento na DZUP envolvem cerca de 20 gêneros de formigas e 14 pesquisadores formados ou em formação, entre projetos de iniciação científica, dissertações de mestrado e teses de doutorado, a saber: Prionopelta (Natalia Ladino), Neocerapachys e Syscia (Paloma Andrade), Gnamptogenys (Weslly Franco, Gabriela Camacho e John Lattke), Cephalotes e Probolomyrmex (Aline Oliveira), Pheidole (Alexandre Casadei-Ferreira), Nomamyrmex (Jaqueline Paes), Ochetomyrmex (Tainara Jory), Kalathomyrmex (Ana Carolina Neundorf), Dinoponera e Hypoponera (Amanda Dias), Neoponera (Adrian Troya), Pachycondyla (Frederico Marcineiro), Strumigenys (Thiago Silva), Leptogenys (John Lattke) Acanthoponera, Camponotus, Centromyrmex e Heteroponera (Rodrigo Feitosa). Não estão inclusos projetos de revisão taxonômica conduzidos em outras instituições, mas que têm o Dr. Rodrigo Feitosa ou o Dr. John Lattke como coorientadores.

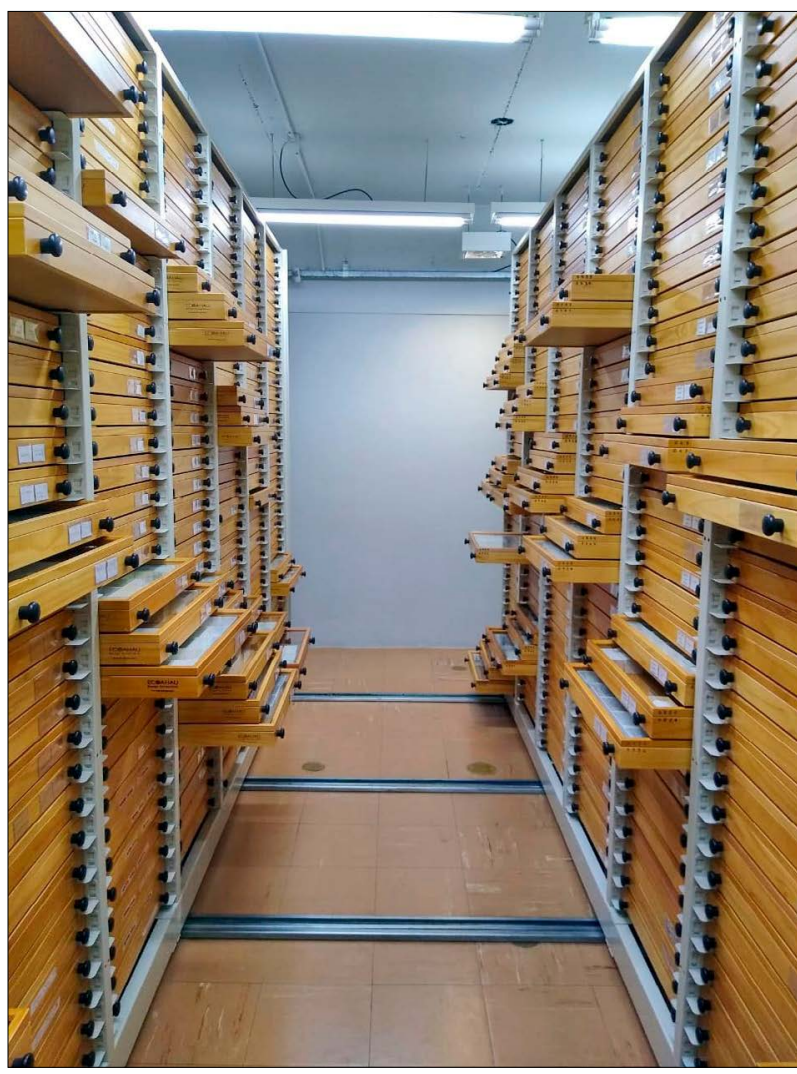

Figura 2. Acervo mirmecológico atual da Coleção Entomológica Padre Jesus Santiago Moure (DZUP), situada na Universidade Federal do Paraná, Curitiba, Brasil. Foto: Paloma Andrade (2019).

Em adição, tais especialistas tendem a descrever com frequência novas espécies que majoritariamente terão seus tipos depositados na DZUP. Assim, embora modesta e recente, esta coleção de formigas tende a crescer exponencialmente em um futuro próximo, consolidando-se como referência entre pesquisadores do Brasil e do exterior.

\section{REFERÊNCIAS}

ALMEIDA, L. M., C. S. RIBEIRO-COSTA \& L. MARINONI, 1998. Manual de coleta, conservação, montagem e identificação de insetos: 1-78. Holos Editora, Ribeirão Preto.

ANDRADE, P. L., 2017. Como nasce uma coleção entomológica? Origem e representatividade do acervo de formigas da Coleção Padre Jesus Santiago Moure. Relatório de Iniciação Científica. Universidade Federal do Paraná, Curitiba.

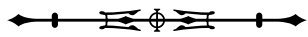


BACCARO, F. B., R. M. FEITOSA, F. FERNANDEZ, I. O. FERNANDES, T. J. IZZO, J. L. P. SOUZA \& R. SOLAR, 2015. Guia para os gêneros de formigas do Brasil: 1-388. Editora INPA, Manaus.

BOLTON, B., 2019. AntCat: an online catalog of the ants of the world. Disponível em: http://www.antcat.org/. Acesso em: 19 dezembro 2019.

CALIXTO, J. M., 2016. Avaliação dos padrões de atividades de formigas de serapilheira (Hymenoptera: Formicidae) nos períodos diurno e noturno. Dissertação (Mestrado em Entomologia) - Universidade Federal do Paraná, Curitiba.

CAMARGO, A. J. A., 2005. A importância de uma coleção entomológica para o país e para o agronegócio em particular. Página Rural, 7 set. 2005. Disponível em: http://www.paginarural.com.br/ artigo/1165/. Acesso em: 19 janeiro 2020.

CAMARGO, A. J. A., 2009. Coleções zoológicas: importância estratégica para o país e para o agronegócio em particular. Portal do Agronegócio, 22 out. 2009. Disponível em: https://www.infoteca. cnptia.embrapa.br/infoteca/bitstream/doc/657316/1/art004.pdf. Acesso em: 19 janeiro 2020.

CAMARGO, A. J. A, C. M OliveirA, M. R. FRIZZAS, K. C. SONODA \& D. C. V. CORRÊA, 2015. Coleções entomológicas: legislação brasileira, coleta, curadoria e taxonomia para as principais ordens: 1-118. EMBRAPA, Brasília.

CARVALHO, C. J. B. \& E. A. B. ALMEIDA, 2011. Biogeografia da América do Sul: padrões e processos: 1-320. Roca, São Paulo.

CONSELHO NACIONAL DE DESENVOLVIMENTO CIENTÍFICO E TECNOLÓGICO (CNPq), 2019. Disponível em: http://www. cnpq.br/. Acesso em: 9 novembro 2019.

FEITOSA LAB, 2018. Laboratório de Sistemática e Biologia de Formigas (UFPR). Disponível em: https://www.feitosalab.com/. Acesso em: 6 novembro 2019.

FRANCO, W., 2018. Riqueza e diversidade de formigas (Hymenoptera: Formicidae) nos campos naturais paranaenses. Dissertação (Mestrado em Entomologia) - Universidade Federal do Paraná, Curitiba.

FRANCO, W., N. LADINO, J. H. DELABIE, A. DEJEAN, J. ORIVEL, M. FICHAUX, S. GROC, M. LEPONCE \& R. FEITOSA, 2019. First checklist of the ants (Hymenoptera::Formicidae) of French Guiana. Zootaxa 4674(5): 509-543. DOI: http://dx.doi.org/10.11646/ zootaxa.4674.5.2.

KEMPF, W. W., 1972. Catálogo abreviado das formigas da região Neotropical. Studia Entomologica 15(1-4): 3-344.

KLINGENBERG, C. \& C. R. F. BRANDÃO, 2005. The type specimens of fungus growing ants, Attini (Hymenoptera, Formicidae, Myrmicinae) deposited in the Museu de Zoologia da Universidade de São Paulo, Brazil. Papéis Avulsos de Zoologia 45(4): 41-50. DOI: http://dx.doi.org/10.1590/S0031-10492005000400001.
LASMAR, C. J., C. R. RIBAS, J. N. C. LOUZADA, A. C. M. QUEIROZ, R. M. FEITOSA, M. M. G. IMATA, G. P. ALVES, G. B. NASCIMENTO, F. S. NEVES \& D. Q. DOMINGOS, 2020. Disentangling elevational and vegetational effects on ant diversity patterns. Acta Oecologica 102: 103489. DOI: https://doi. org/10.1016/j.actao.2019.103489.

LOYOLA E SILVA, J., 1992. Padre Jesus Santiago Moure - 80 anos. Revista Brasileira de Zoologia 9(1-2): 1. DOI: http://dx.doi. org/10.1590/S0101-81751992000100001.

MARINONI, R. C. \& R. R. C. DUTRA, 1991. Levantamento da fauna entomológica no estado do Paraná. I. Introdução. Situações climática e florística de oito pontos de coleta. Dados faunísticos de agosto de 1986 a julho de 1987. Revista Brasileira de Zoologia 8(1-4): 31-73. DOI: https://doi.org/10.1590/S0101-81751991000100005.

MARINONI, R. C., L. M. ALMEIDA, D. S. NAPP \& G. H. ROSADONETO, 1992. Primeira lista do material-tipo de Coleoptera da Coleção de Entomologia Pe. J.S. Moure, do Departamento de Zoologia da Universidade Federal do Paraná. Revista Brasileira de Zoologia 9(1-2): 99-126. DOI: http://dx.doi.org/10.1590/S010181751992000100012

MARINONI, L. \& R. G. MELO, 2020. DZUP- Hymenoptera Coleção Entomológica Pe. Jesus Santiago Moure (Hymenoptera). Version 1.19. Universidade Federal do Paraná, Curitiba. DOI: https:// doi.org/10.15468/cwlwvm.

MARTINS, M. F. O., M. J. THOMAZINI, D. BARRETA, G. G. BROWN, M. G. ROSA, M. R. G. ZAGATTO, A. SANTOS, H. S. NADOLNY, G. B. X. CARDOSO, C. C. NIVA, M. L. C. BARTZ \& R. M. FEITOSA, 2020. Accessing the subterranean ant fauna (Hymenoptera: Formicidae) in native and modified subtropical landscapes in the Neotropics. Biota Neotropica 20(1): e20190782. DOI: http://dx.doi.org/10.1590/1676-0611-bn-2019-0782

MELO, M. S., G. B. GUIMARÃES, A. F. RAMOS \& C. C. PRIETO, 2007. Relevo e hidrografia dos Campos Gerais. In: M. S. MELO, R. S. MORO \& G. B. GUIMARÃES (Ed.): Patrimônio natural dos Campos Gerais do Paraná: 93-98. Editora UEPG, Ponta Grossa, Paraná.

MOURE, J. S., D. URBAN \& G. A. R. MELO (Org.), 2007. Catalogue of bees (Hymenoptera, Apoidea) in the neotropical region: 1-1058. Sociedade Brasileira de Entomologia, Curitiba.

OLIVEIRA, R. H. R., 1994. A reforma agrária e suas implicações no processo de desenvolvimento do estado de Rondônia. Dissertação (Mestrado em Administração Pública) - Fundação Getúlio Vargas, Rio de Janeiro.

OLIVEIRA, A. M., 2015. Composição da fauna de formigas no extremo meridional do bioma cerrado. Relatório de Iniciação Científica. Universidade Federal do Paraná, Curitiba.

RAFAEL, J. A., G. A. R. MELO, C. J. B. CARVALHO, S. A. CASARI \& R. CONSTANTINO (Ed.)., 2012. Insetos do Brasil: diversidade e taxonomia: 1-795. Holos, Ribeirão Preto.

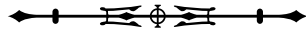


SPECIESLINK, 2019. Disponível em: http://splink.cria.org.br/. Acesso em: 18 outubro 2019.

TAXONLINE, 2019. Rede paranaense de coleções biológicas. Disponível em: http://taxonline.bio.br/. Acesso em: 2 novembro 2019.

ULYSSÉA, M. A., C. E. CERETO, F. B. ROSUMEK, R. R. SILVA \& B. C. LOPES, 2011. Updated list of ant species (Hymenoptera, Formicidae) recorded in Santa Catarina State, southern Brazil, with a discussion of research advances and priorities. Revista Brasileira de Entomologia 55(4): 603-611. DOI: http://dx.doi.org/10.1590/ S0085-56262011000400018.
UNIVERSIDADE FEDERAL DO PARANÁ (UFPR), 2019. Departamento de Zoologia. Disponível em: http://www.bio.ufpr. br/portal/zoologia/. Acesso em: 10 novembro 2019. 